computerized technique. The hypothesis is supported by the observation that one newly arrived cosmonaut on the Russian space station MIR, upon dropping his camera, immediately reached "down" to grab it, missing completely as the camera continued moving away in an unanticipated straight line.

If the constant-gravity model is supported by this experiment, the investigation will focus on whether and how the response changes over the mission duration. On the other hand, if subjects perform perfectly in microgravity from the beginning, follow-up experiments during future shuttle flights are planned to determine whether calculation of the gravitational force is based on visual, vestibular, proprioceptive information or on efferent copy from motor commands. These experiments will rely on a virtual environment, which allows visual and vestibular cues to be manipulated independently. The virtual environment generator includes a three-dimensional graphics workstation, helmet-mounted display, head tracker and joystick. The subject also wears a harness that pulls the body "downward" to simulate gravity. This apparatus gives the experimenters more complete control of the sensory input than would be possible on earth.

Aside from producing temporary adaptations in the spatial computations of adult animals, the sensory environment has profound and often permanent effects on neural development. For example, visual experience during a critical period is required to produce the normal pattern of connections from the thalamus to the visual cortex, and changes in visual input can cause permanent deficits in young animals. If neural activity is similarly important for developing vestibular areas, then the signals provided by gravity are expected to be critical for normal synapse formation in brain regions that process spatial information.

Ken Kosik at Harvard and Oswald Steward at the University of Virginia are testing this by investigating the effects of gravity on synapse development. Young rats are raised in microgravity from postnatal day 4 to 20 days of age. This period was chosen because synapse number, dendritic complexity and spine number, and expression of synaptic proteins all increase dramatically in the hippocampus during this time. Upon returning from space, the animals will be perfused for light and electron microscopy. Because of space shuttle procedures, the earliest time point is approximately 24 hours after landing, and the second is at 30 days after landing to assay for long-lasting changes. Synapse development in several brain regions will be determined with unbiased stereological sampling to determine volume and synapse density.

Three regions have been chosen for analysis. The vestibulocerebellum receives input from vestibular nuclei and sends an inhibitory projection to brainstem motor pathways thought to be important for posture and balance. The parietal cortex receives information from sensory association areas and projects to motor cortex. As discussed above, the hippocampus signals location in space and responds to vestibular information. Given that many other sensory systems show experiencedependent plasticity during development, it is possible that any or all of these regions may show alterations as a result of the exposure to microgravity.
There is of course much uncertainty as to whether any of the Neurolab experiments will work exactly as planned. They have been thoroughly screened for feasibility, with extensive pilot experiments culminating in dummy runs performed by the astronauts themselves in a mock-up of the shuttle lab. But there is plenty that could still go wrong; McNaughton notes, for instance, that although his rats are trained and the tetrodes are already implanted and working (two weeks prior to the launch date), the effects of brain edema in zero gravity may yet compromise the recordings once the rats are in space. Worse still, the rats may decide on exposure to zero gravity that the novelty of floating round the cabin outweighs the rewards of cooperation with the experimenters. But these uncertainties are inescapable; it is of course the unfamiliarity of the zero gravity environment that constitutes the justification, as well as the risk, of all the Neurolab experiments.

\title{
Listen up
}

With their highly developed sense of hearing, bats hold a special fascination for auditory physiologists. On page 54, Yan \& Suga examine the role of corticofugal projections in the auditory system in the big brown bat (shown here). As with other mammals, sound stimuli reach the auditory cortex by way of the inferior colliculus (IC) of the midbrain, which in turn receives a large feedback projection from the cortex. The connections between the two areas are tonotopic; the IC contains a map of auditory frequencies, which projects via the thalamus to form a corresponding map in the cortex. But the significance of the reciprocal connections from the cortex to the IC (the corticofugal projection, a common feature of sensory systems) is less well understood. The authors have investigated this question by stimulating the cortex, either electrically or with sounds, and examining the effect on the frequency-selective neurons of the IC. Stimulating the cortex at a given frequency causes shifts in the tuning of IC neurons in the corresponding frequency range. The net effect of these shifts, which persist for at least 30 minutes, is to enhance the midbrain response to the stimulated frequency, while suppressing responses to adjacent frequencies. The authors suggest that one function of the corticofugal system may be to modulate the lower levels of the auditory system in response to auditory experience.

Charles Jennings 